\title{
DESENVOLVIMENTO DA LINGUAGEM EM SITUAÇÕES DE INTERAÇÃO SOCIAL COM GRUPOS MINORITÁRIOS: ENTREVISTA COM CÉLIA RENATA ROSEMBERG
}

\author{
Por: Gabriela Medeiros Nogueira \\ Universidade Federal do Rio Grande- FURG
}

Em 2008, tive o privilégio de ser recebida pela professora Célia Renata Rosemberg, na Facultad de Filosofía y Letras da Universidad de Buenos Aires, para realizar o doutorado sanduiche no âmbito do Programa de Fortalecimiento Del Doctorado UPFEL - UBA. Desde então, desenvolvemos projetos de pesquisa em parceria, e realizamos missões de estudo a curto prazo, com intercâmbio entre pesquisadores.

Célia é professora titular de "Investigación y Estadística Educacional I" na Facultad de Filosofía y Letras, na Universidad de Buenos Aires. Desde 2016, atua como diretora do "Centro Interdisciplinario de Investigacionesen Psicología Matemática y Experimental (CIIPME)". E ainda, é investigadora do "Consejo Nacional de Investigaciones Científicas y Técnicas", com uma longa trajetória de pesquisa no campo da alfabetização. Conta com uma vasta produção de artigos científicos publicados em âmbito nacional e internacional, de livros acadêmicos e também livros para crianças, visando o desenvolvimento da linguagem. Vários desses livros são bilíngues, ou seja, são escritos em espanhol e outra língua, geralmente de origem indígena. Cabe destacar, que parte de sua investigação sobre alfabetização, é realizada em comunidades étnicos minoritárias, tais como, Tobas e Qom de Chaco e Collas del Noroeste Argentino e também com grupos de pessoas em situação de vulnerabilidade social. Muito do material produzido pela professora Célia e sua equipe de pesquisadores, procura dar visibilidade às crianças, tornando-as personagens protagonistas dos livros, sendo que a linguagem do seu cotidiano e o vocabulário próprio fazem parte dos diálogos das histórias. Temáticas como alfabetização multicultural, alfabetização bilíngue, interculturalidade, são recorrentes em suas produções, assim como, narração e leitura de contos, compreensão da leitura, vocabulário e intercâmbio verbal.

Outro aspecto relevante a ser destacado em relação à temática investigativa e a proposta de ação promovida pela equipe de pesquisa, coordenada por Célia, é sobre "niños tutores e na alfabetización". Trata-se de um projeto em que crianças mais velhas auxiliam as mais novas, tanto na escola como em casa, lendo histórias, promovendo a interação verbal e desenvolvimento linguístico. Nesta mesma linha, a equipe da professora Célia realiza um trabalho voltado para a comunidade, promovendo oficinas para famílias.

Diante da relevância e dimensão de seu trabalho, citado resumidamente nesta apresentação, considero que a entrevista que segue contribui imensamente com a temática deste dossiê, bem como para ampliar o diálogo com alfabetizadores e fortalecer a interlocução entre pesquisadores da América Latina. 
Entrevistadora - Poderias explicar o que é o CONICET e, especialmente, relatar sobre o trabalho que desenvolves neste centro de investigação?

Célia Rosemberg -El CIIPME, (Centro Interdisciplinario de Investigaciones en Psicología Matemática y Experimental que es el Instituto de investigación en el que yo trabajo (y que actualmente dirijo) es un centro de investigación que depende directamente del CONICET (Consejo Nacional de Investigaciones Científicas y Técnicas, Argentina).

EI CONICET es un organismo gubernamental que sostiene y promueve la ciencia y la tecnología en Argentina y que fue creado en 1958. Es una entidad autárquica que depende del Ministerio de Ciencia y Tecnología. El objetivo de CONICET es fomentar e implementar actividades científicas y tecnológicas en todo el territorio argentino y en todas las áreas de conocimiento. Actualmente posee una planta de más de 10000 investigadores, además de 11000 becarios doctorales y postdoctorales y 2600 técnicos y personal profesional de apoyo a la investigación

El CIIPME se encuentra ubicado en el centro de la Ciudad de Buenos Aires, y allí trabajan alrededor de 60 personas, entre investigadores, becarios y personal de apoyo a la investigación. Las investigaciones del Instituto se centran en el desarrollo cognitivo, lingüístico, socioemocional y neuropsicológico, y analizan aspectos sociales de la experiencia humana que se manifiestan a través del desarrollo en distintos contextos culturales.

El grupo de investigación "Lenguaje, cognición e interacción social en la infancia" que es el que yo coordino, constituye hoy en día uno de los programas de investigación más numerosos del CIIPME y reúne una serie de proyectos de investigación que estudian el desarrollo del lenguaje en la infancia. Los proyectos se focalizan en el desarrollo del lenguaje oral -vocabulario, gramática y discurso narrativo y argumentativo- y en la alfabetización temprana. La mayor parte de nuestros estudios atienden a estos desarrollos en el marco de los contextos de interacción en los que se producen en el hogar, la comunidad y el sistema escolar.

Entrevistadora - Tens um reconhecido trabalho investigativo no campo da alfabetização desde a década de 90. Poderias explicar sobre a perspectiva teórica, metodológica, sobre os sujeitos, e os aspectos das pesquisas que desenvolves nesse campo da alfabetização?

Célia Rosemberg - Las investigaciones de mi grupo parten de una perspectiva psicolingüística que otorga a la interacción social un rol relevante. Articula el enfoque sociocultural del desarrollo humano (Vygotsky, 1964), con los modelos psicolingüísticos actuales (Nelson, 1996, 2007; Tomasello, 1998, 2003) y con conceptos de la sociolingüística interaccional (Gumperz, 1982, 1984) y el análisis conversacional (Schegloff, 1979; Schegloff, Jefferson y Sacks, 1977; Goodwin, 2000; Goodwin, 2003).

Desde esta perspectiva asumimos que el desarrollo del lenguaje tiene lugar en contextos de interacción en los que se lo usa tempranamente en la producción discursiva para llevar a cabo actividades compartidas. En términos generales, consideramos que el proceso de adquisición del lenguaje, al igual que otros aprendizajes socioculturales, involucra un cierto desarrollo y preparación sociocognitiva por parte del niño. Los niños se apoyan en sus capacidades para percibir, categorizar y conceptualizar asì como en las capacidades que poseen para establecer relaciones intersubjetivas con otras personas y centrar su atención de modo conjunto con ellas en aspectos determinados del contexto de la situación de interacción. Pero la adquisición del lenguaje requiere, además, del sostén de compañeros culturales (pares y adultos) que andamien la experiencia infantil con los objetos, los conocimientos y los instrumentos con los que se encuentran en las actividades de las que el niño participa. 
Para poder dar cuenta de estas dimensiones situacionales, discursivas, lingüísticas y cognitivas que impactan en el desarrollo y el aprendizaje infantil recurrimos a estrategias metodológicas mixtas. Estas estrategias combinan una aproximación ecológica a las situaciones de interacción en las que niños y niñas participan y la identificación, descripción y ponderación de los factores que puedan afectar sus oportunidades de desarrollo del lenguaje y la alfabetización temprana: estrategias cualitativas para abordar de modo situado las interacciones espontáneas verbales y no verbales; la cuantificación de ciertas variables específicas que caracterizan estas interacciones y el empleo de estrategias cuasi experimentales que generen contextos semiestructurados en los que sea pasible la observación de ciertos fenómenos particulares.

Entrevistadora- Um dos aspectos que abordas em tuas pesquisas é sobre a narrativa no processo de alfabetização. Gostaríamos de saber um pouco mais sobre esse aspecto.

Célia Rosemberg - En el marco de estos modelos teóricos en los que nosotras desarrollamos nuestras investigaciones se considera que la narración no es solo una forma de discurso que organiza los intercambios comunicativos, sino también un esquema cognitivo, una de las primeras y más importantes formas en que se configuran el pensamiento y la memoria. La narración es el modo natural en el que las categorías lingüísticas se imponen -organizan y representan-a las estructuras conceptuales derivadas de la experiencia. El discurso narrativo refleja las secuencias de acciones en las que todos estamos insertos en nuestra vida cotidiana.

Desde su nacimiento, los niños y niñas participan de situaciones de juego, comida e higiene compartidas con los miembros de su familia y de su comunidad. Todas estas situaciones responden a una estructura narrativa: constituyen secuencias de acciones llevadas a cabo por individuos que intentan alcanzar ciertos objetivos. A partir de la participación frecuente y reiterada en estas rutinas cotidianas, los niños construyen esquemas mentales que representan estas secuencias de acciones, que tienen un formato narrativo. El uso de estos esquemas mentales le permite al niño progresivamente entender lo que sucede, actuar en las situaciones, recordar lo que ha sucedido y anticipar lo que sucederá. Es decir, la narrativa es una forma de pensamiento que permite comprender la realidad, construir, a partir de los recuerdos más importantes la memoria autobiográfica que es la base para el desarrollo de la identidad individual y comunitaria y actuar sobre la realidad para transformarla.

Entrevistadora - Tens trabalhado também com a produção de materiais pedagógicos. Poderias relatar como vens elaborando este trabalho e como se articula com as investigações que desenvolves. A elaboração destes materiais tem a participação de professores que estão diretamente na escola? E as crianças participam de alguma forma?

CéliaRosemberg - La línea de investigación que desarrollé junto con mi equipo se caracteriza por estudiar el lenguaje en uso, esto es, el lenguaje en los contextos naturales de interacción en los que los niños y niñas espontáneamente participan. Con una aproximación ecológica observamos y registramos las situaciones cotidianas en los hogares, las comunidades y la escuelas. A partir de esos registros estudiamos los modos de habla, la cantidad de palabras que los chicos y chicas escuchan en sus entornos, el vocabulario, cuántas palabras se dicen, el estilo discursivo, para qué se narra, para qué se argumenta, los recursos lingüísticos, cuáles son las formas discursivas que se ponen en juego. Comparamos, también, lo que los chicos de sectores más pobres escuchan en términos de lenguaje con lo que escuchan los chicos de sectores medios, qué narrativas producen unos y otros, qué los mueve a narrar y si esas formas de lenguajes son o no retomadas por la escuela. 
A partir de los resultados de esos trabajos nosotras nos propusimos capitalizar los conocimientos usos del lenguaje y patrones de interacción que eran propios de la comunidad de los niños para enseñarles otras cosas y ampliar su mundo. Nosotras basamos las propuestas de intervención en las características socioculturales de la población y en los patrones de interacción y en los conocimientos y en el capital cultural y lingüísticos que observamos que tienen los distintos grupos sociales. Desarrollamos tanto módulos de formación docente como libros de lectura para los niños. Ello lo llevamos a cabo como un modo de poder transferir directamente a los chicos y chicas los resultados de nuestra investigación. El primero de estos libros es "Las Aventuras de Ernestina" que elaboramos a partir de la investigación en las comunidades collas del Noroeste argentino. Como voy a señalar con relación a la elaboración de ese libro, los docentes participan activamente junto a los investigadores en todo el proceso.

Entrevistadora - No final da década de 90 vocês desenvolveram uma pesquisa com "niños de las comunidades colas del Noroeste Argentino". Poderias tratar sobre esta pesquisa, principalmente sobre o que motivou este trabalho? Teve apoio financeiro? Quem participou? Como foi realizada? Quais os desdobramentos?

Célia Rosemberg - Se trató de una investigación que realizamos con Ana María Borzone en las comunidades collas en colaboración con una organización social de la zona de Humahuaca: el programa Yachay de la Fundación OCLADE (Rosemberg y Borzone, 1998; Borzone y Rosemberg, 2000) y financiada por la Fundación Bernard Van Leer de Holanda. El estudio consistió en un diagnóstico en profundidad de los factores que se relacionaban con las dificultades que los chicos collas manifestaban para aprender a leer y escribir en la escuela.

Cuando comenzamos la investigación nosotras ya sabíamos, por los estudios previos, que todos los niños y niñas tienen una gran potencialidad para aprender y que no tienen ningún déficit lingüístico ni cognitivo que sea un impedimento para el dominio de la lectura y la escritura. ¿Dónde buscar entonces indicios que nos permitieran comprender las dificultades que mostraban los niños? Orientadas por las investigaciones en la línea de la psicología sociocultural y de la sociolingüística interaccional decidimos desplazar el foco de la consideración del niño en forma aislada a considerar a los chicos en el marco de la compleja interacción entre sus entornos fundamentales: el hogar, la comunidad y la escuela. De ahí que elaboramos un diseño comparativo en el que confrontábamos las situaciones de interacción en las que los chicos participaban en estos tres ámbitos. Trabajamos en 4 comunidades: dos de Jujuy -Suripugio y Paicone- y dos de Salta - Pueblo Viejo y Poscaya-. En las comunidades filmamos las clases en la escuela y en salitas comunitarias de educación inicial y realizamos el seguimiento de 16 niños, 4 de cada comunidad, en sus interacciones en el hogar y la comunidad.

La recolección y el análisis de la información empírica fue realizada en forma conjunta por el equipo de investigación: los promotores del programa y nosotras como coordinadoras técnicas. Los promotores se instalaban por períodos de 3 días en las comunidades y seguían a los niños en todos sus juegos e interacciones en la comunidad. Para poder registrar todos los intercambios lingüísticos en los que los chicos y chicas participaban, les colgaban del cuello un grabador. Los videos de las escuelas y las grabaciones de los chicos en sus casas y en sus comunidades fueron transcriptos en forma muy detallada y respetando las características fonológicas, sintácticas y discursivas del dialecto hablado en las comunidades. 
Los resultados del análisis comparativo del desempeño lingüístico y cognitivo de los niños en el hogar, la comunidad y la escuela nos mostró que la interacción entre las prácticas de enseñanza escolares y una fractura muy marcada entre el hogar y la escuela en cuanto a las formas de intercambio verbal, los usos del lenguaje, los conocimientos y el estilo de aprendizaje, podía proporcionar una explicación a las dificultades que tienen los chicos collas en sus aprendizajes escolares. A partir de esta investigación tomé conciencia de que es necesaria una escuela que pueda prestar especial atención a estas diferencias y pensar distintas estrategias a fin de lograr que la comunicación y, por ende, la enseñanza, sean efectivas. Puede parecer paradójico plantear un mismo objetivo para todos: un alto nivel de alfabetización, y no plantear la misma escuela para todos. La única vía para alcanzar igualdad en los resultados es que la intervención pedagógica parta del mundo social del niño y apoye la construcción de nuevos conocimientos vinculándolos con su contexto de referencia.

Nos preguntamos, entonces, cómo partir del capital cultural y lingüístico de los chicos, cómo hacer para contribuir a superar la fractura tan marcada entre el hogar y la escuela, qué estrategia emplear para incluir en el interior del proceso de enseñanza y aprendizaje el habla, las costumbres, la cotidianeidad de los chicos que pertenecen a grupos sociales que no comparten el dialecto y la cultura de la escuela. Este fue el origen del libro Las aventuras de Ernestina.

"Las aventuras de Ernestina" es un resultado directo de la investigación en las comunidades collas. Ernestina, la protagonista, es una de las niñas que grabamos durante días y días en su comunidad Pueblo Viejo (Salta). Junto con Eulalia Flores, la maestra y promotora del programa que realizó sus observaciones y con Ana María Borzone escribimos el libro partiendo de los registros de observación y novelando los episodios. No se trata simplemente de un libro que recupera temas, conocimientos y problemáticas locales, sino que se trata de un libro escrito en base a observaciones de la vida cotidiana de los chicos y es, en este sentido, propiamente etnográfico. Recupera la mirada de los chicos como actores en esa realidad, sus intereses e interacciones en relación con los objetos, las personas y los problemas de su comunidad. Refleja la cultura de los niños, su perspectiva de la vida, pero, a su vez, los introduce en otras formas culturales. Es por ello, un libro intercultural. Asimismo, es bidialectal, ya que mientras que el narrador emplea el dialecto estándar, en las transcripciones de los diálogos de Ernestina y de otros miembros de la comunidad aparece el dialecto colla.

Entrevistadora - E os livros infantis "Enla casa de Oscarcito"? O que são? Como foram elaborados e qual a repercussão deste trabalho?

CéliaRosemberg - Los libros "En la casa de Oscarcito" son parte del proyecto "Oscarcito" en el que se elaboró, implementó y evaluó un programa para ampliar las oportunidades desarrollo infantil de chicos(as) de 3 a 5 años que viven en barrios de extrema pobreza de Buenos Aires. El programa lleva a cabo acciones educativas en los hogares de los chicos y en los jardines de infantes comunitarios a los que asisten. Del mismo modo que todas nuestras otras propuestas pedagógicas estas acciones, especialmente diseñadas, recuperan los conocimientos y el lenguaje de los chicos y buscan, a partir de allí, ampliar su vocabulario, su conocimiento del mundo, sus estrategias de discurso, el aprendizaje de la variedad lingüística estándar y el ingreso al proceso de alfabetización. 
Empezamos a diseñar el proyecto en el 2003 y en el 2004, con el apoyo de Elke Rottgardt y Horst Schroth, que vía la Fundación Care de Alemania, financiaron todo el proceso de intervención. Con su apoyo, pudimos comenzar a realizar la implementación en la Ciudad y el conurbano de Buenos Aires. Posteriormente con el apoyo de la Fundación Arcor, y a través de un convenio con el Ministerio de Educación de la Provincia de Entre Ríos la implementación alcanzó a todos los niños y niñas de las salas de 5 años de la Provincia de Entre Ríos.

Los libros En la casa de Oscarcito están pensados para ser usados específicamente con las familias. En nuestro país no hay otros programas que incorporen a las familias. Nosotras trabajamos con ellas, les entregamos los libros y les mostramos estrategias para promover el desarrollo del lenguaje en los chicos.

Entrevistadora - Como as línguas minoritárias podem influenciar a alfabetização na Argentina? Como as práticas alfabetizadoras têm lidado com a interculturalidade a multiculturalidade?

Célia Rosemberg - Mi equipo de investigación trabaja actualmente con la comunidad Qom/ Toba que es originaria del noroeste de nuestro país. En particular nosotras trabajamos en la Provincia de Chaco. Se trata de una lengua que se halla en riesgo, dado que está siendo reemplazada por el español en ciertos contextos comunicativos. Es por ello que, desde una perspectiva sociolingüística, resulta relevante orientar esfuerzos educativos y de investigación hacia el fortalecimiento de la lengua.

Como en todos nuestros otros proyectos, también en este caso articulamos acciones de investigación en torno al desarrollo lingüístico y acciones educativas en la comunidad indígena. Estas acciones dieron lugar a la creación de un corpus empírico de situaciones de interacción de las que participan los niños y niñas en sus hogares y en la comunidad. Este corpus sirve como (i) base para una serie de estudios sobre la adquisición del lenguaje, y (ii) un recurso para acciones educativas dirigidas a ampliar las oportunidades de desarrollo de lo

s/as niños/as y a fortalecer la vitalidad de la lengua qom.

Desarrollamos un programa de alfabetización bilingüe e intercultural que, recupera los conocimientos y la lengua que los/as niños/as experimentaron en su socialización primaria. El programa atiende a las diferencias culturales y lingüísticas entre las comunidades qom ubicadas en distintas regiones y con diferente grado de urbanización, hibridización de sus prácticas culturales y situaciones sociolingüísticas. Así, Junto con Gladys Ojea, Florencia Alam, y un nutrido grupo de docentes indígenas y miembros de las comunidades diseñamos una serie de libros de lectura etnográficos en qom y en español. Estos libros constituyeron una de las principales acciones para incluir en la escuela el lenguaje y los conocimientos que caracterizan al ámbito familiar y comunitario.

Los libros fueron escritos a partir de las observaciones realizadas en el trabajo de campo en colaboración con los docentes indígenas y miembros de la comunidad. Los libros permiten la alfabetización bilingüe en qom y español, comenzando con la lengua predominante en la socialización primaria del niño/a y luego iniciando la alfabetización en la segunda lengua, a la vez que se refuerza su dominio oral. En Aso nogotolele' enaxat Cintia [Una niña llamada Cintia], la protagonista es una niña de una comunidad rural hablante nativa de qom. El libro intercultural en español Las Aventuras de Huaqajñe se basa en las observaciones de una niña qom de zona semi-urbana que tiene como primera lengua el español. Los libros de historias en imágenes para la enseñanza del qom y del español como segunda lengua: Dany y Dany: Na'aqtaguec, Na'qaatqa, Relatos, Tolhomtes [Dany: Relatos] están basados en las observaciones de Daniel, un niño bilingüe de zona rural. 
También los libros bilingües, En la Comunidad Daviaxaiqui (La Familia de Ana, El Abuelo Valentín, Los Nietos de Ramón) para niños/as de una comunidad de migrantes en Buenos Aires, promueven la revitalización de la lengua dado que, simultáneamente con el español (lengua que los niños dominan), introducen el qom como segunda lengua a través de historias y actividades de vocabulario. En las diferentes comunidades en las que trabajamos también se llevaron a cabo instancias de desarrollo profesional docente.

Entrevistadora - Agradeço imensamente a professora Célia Renata Rosemberg por conceder esta entrevista e compartilhar aspectos de sua trajetória profissional. O relato da professora sobre os aspectos teóricos e metodológicos que seu grupo de pesquisa desenvolve na Argentina, possibilitam a reflexão sobre como realizamos nossas pesquisas no campo da alfabetização no Brasil.

Recebido em: 15/01/2020

Aceito em: 25/03/2020 